\title{
Wwp2 is essential for palatogenesis mediated by the interaction between Sox9 and mediator subunit 25
}

\author{
Yukio Nakamura, ${ }^{1, \star}$, Koji Yamamoto ${ }^{2, \star}$, Xinjun He³, Bungo Otsuki², Youngwoo Kim², Hiroki Murao², \\ Tsunemitsu Soeda ${ }^{2}$, Noriyuki Tsumaki ${ }^{4}$, Jian Min Deng ${ }^{5}$, Zhaoping Zhang ${ }^{5}$, Richard R. Behringer ${ }^{5}$, \\ Benoit de Crombrugghe ${ }^{5}$, John H. Postlethwait ${ }^{3}$, Matthew L. Warman', Takashi Nakamura² \& Haruhiko Akiyama²
}

Sox9 is a direct transcriptional activator of cartilage-specific extracellular matrix genes and has essential roles in chondrogenesis. Mutations in or around the SOX9 gene cause campomelic dysplasia or Pierre Robin Sequence. However, Sox9-dependent transcriptional control in chondrogenesis remains largely unknown. Here we identify Wwp2 as a direct target of Sox9. Wwp2 interacts physically with Sox 9 and is associated with Sox 9 transcriptional activity via its nuclear translocation. A yeast two-hybrid screen using a cDNA library reveals that Wwp2 interacts with Med25, a component of the Mediator complex. The positive regulation of Sox 9 transcriptional activity by Wwp2 is mediated by the binding between Sox 9 and Med25. In zebrafish, morpholino-mediated knockdown of either wwp2 or med25 induces palatal malformation, which is comparable to that in sox 9 mutants. These results provide evidence that the regulatory interaction between Sox9, Wwp2 and Med25 defines the Sox 9 transcriptional mechanisms of chondrogenesis in the forming palate.

\footnotetext{
Clinical Research Center, Murayama Medical Center, Tokyo 208-0011, Japan. ${ }^{2}$ Department of Orthopaedics, Kyoto University, Kyoto 606-8507, Japan. ${ }^{3}$ Institute of Neuroscience, University of Oregon, Eugene, Oregon 97403, USA. ${ }^{4}$ Department of Bone and Cartilage Biology, Osaka University, Osaka 565-0871, Japan. ${ }^{5}$ Department of Genetics, The University of Texas M.D. Anderson Cancer Center, Houston, Texas 77030, USA. ${ }^{6}$ Howard Hughes Medical Institute, Department of Orthopaedic Surgery and Genetics, Children's Hospital and Harvard Medical School, Boston, Massachusetts 02115, USA . ${ }^{\star}$ These authors contributed equally to this work. Correspondence and requests for materials should be addressed to H.A. (email: hakiyama@kuhp.kyoto-u.ac.jp).
} 
$\mathrm{T}$ he initiation of mesenchymal condensation and overt chondrogenesis depend on the transcription factor Sox9. Loss-of-function and gain-of-function analyses using mouse genetic approaches have revealed that Sox 9 has an essential role in successive steps of chondrogenesis that are mediated by cell proliferation, differentiation and extracellular matrix production ${ }^{1,2}$. Haploinsufficiency of SOX9 causes campomelic dysplasia, which is a severe and often lethal skeletal dysplasia associated with sex reversal ${ }^{3,4}$. Hypomorphic intragenic mutations or position effect translocation breakpoints around the SOX9 gene cause the siteand stage-specific disruption of SOX9 transcription, resulting in Pierre Robin Sequence ${ }^{5}$, which is characterized by micrognathis, glossoptosis and cleft palate. Indeed, heterozygous Sox 9 mutant mice died perinatally with cleft palate ${ }^{6}$. Previous studies have showed that Sox9 induces L-Sox5 and Sox6 transcription, and these Sox proteins coordinately regulate the expression of cartilage-specific extracellular matrix genes, including Col2a1, Coll1a2 and Aggrecan ${ }^{1,7-9}$. Genetic variants in these genes also cause palatal malformation ${ }^{10-12}$. These findings indicate that Sox 9 transcriptional activity has a regulatory role in palatogenesis. The activity of Sox 9 in chondrogenesis is controlled by several transcription factors. Recently, the thyroid hormone receptor-associated protein 230/Med12 (which is a member of the Mediator complex), the nuclear RNA-binding protein, $\mathrm{p} 54^{\text {nrb }}$ the CREB-binding protein $(\mathrm{CBP}) / \mathrm{p} 300$, and the Tat interactive protein-60 have been reported as modulators of Sox 9 transcriptional activity ${ }^{13-17}$. However, Sox9-related genes and Sox9-dependent transcriptional regulation during chondrogenesis are not fully understood.

To define the repertoire of Sox9-dependent genes that contribute to the regulation of chondrogenesis, we generated Sox $9-3^{\prime}$ enhanced green fluorescent protein (EGFP) knock-in mice and Sox9-EGFP/ EGFP null chimeras, and then performed a fluorescence-activated cell sorting (FACS)/comparative microarray gene expression analysis using EGFP-positive cells derived from limb buds of the mouse embryos. Subsequently, using in situ hybridization analysis of Sox9 in limb buds of wild-type and conditional Sox9 knockout embryos harbouring the Prx1-Cre transgene, we identified genes that may be involved in chondrogenesis. Of these candidate genes, we focused on Wwp2, a member of the neural precursor cell expressed, developmentally downregulated 4-like protein family and the HECT domain E3 ubiquitin-protein ligases ${ }^{18}$. Wwp2 controls the epithelial $\mathrm{Na}(+)$ channel ${ }^{19}$ and the divalent metal ion transporter DMT $1^{20}$. In addition, recent reports have showed that Wwp2 mediates Oct4 ubiquitination and degradation in embryonic stem (ES) cells ${ }^{21}$. However, the expression and functions of Wwp2 in chondrogenesis remain unknown.

Here we demonstrate that Wwp2 interacts physically with Sox9 and Med25, mediator of RNA polymerase II transcription subunit 25 , and reveal that Med25 transmits Sox 9 transcriptional activity, which is mediated by binding with Wwp2. Furthermore, morpholino-mediated knockdown of either wwp2 or med 25 induced palatal malformation in zebrafish, which is comparable to that observed in zebrafish sox9 mutants. These findings provide new insights into the regulatory mechanisms of Sox9 transcriptional activity in chondrogenesis.

\section{Results}

Wwp2 is identified as a Sox9-related gene in chondrogenesis. To define the repertoire of in vivo Sox9-dependent genes in chondrogenesis, we performed FACS on genetically engineered mouse embryos that selectively express EGFP in a Sox9-specific pattern, followed by whole-genome microarray expression profiling on the isolated cells. For this purpose, we generated mutant mice in which an internal ribosome entry site (IRES)-EGFP-pA/loxP-flanked $P G K$-neo-bpA cassette was inserted into the $3^{\prime}$-untranslated region of the Sox 9 gene in exon 3 just at the $5^{\prime}$ end to the polyadenylation signal (Sox9-3' EGFP knock-in mice; Fig. 1a,b). Heterozygous and homozygous Sox9-3' EGFP knock-in mice were viable and fertile and showed no noticeable phenotypic changes. In addition, EGFP expression was detectable in a Sox9-specific pattern during embryogenesis (Fig. 1c). Next, we generated Sox9-EGFP/EGFP null ES cells by insertion of IRES-EGFP-pA/loxP-flanked PGK-neobpA cassette into the coding regions of the Sox9 gene (Fig. 1d-g). Our target strategy functionally inactivated Sox9 and led to the expression of EGFP in a Sox9-specific pattern.

The purity of EGFP-positive cells of Sox9-3' EGFP knock-in and Sox9-EGFP/EGFP null chimeric embryos harvested from limb buds at embryonic day 12.5 (E12.5) was 96.0 and $94.8 \%$, respectively (Fig. 1h), and reverse transcription (RT)-PCR analysis revealed that the expression of Sox9 in the majority of the population of cells isolated from Sox9-EGFP/EGFP null chimeras was deleted (Fig. 1i). Following the first screening of the microarray analysis (the complete annotated data set is available from the ArrayExpress repository, accession number E-MTAB-537), we further narrowed down the Sox9-related candidate genes using in situ hybridization in limb buds of E12.5 wild-type and conditional Sox9 knockout embryos harbouring the Prx1-Cre transgene. With the exception of Sox5, Sox6, Col2a1, Col9a1, Col9a3 and Col11a1, 27 genes that exhibited the same pattern as Sox9 in conditional Sox9 knockout embryos were identified as Sox9-related genes involved in chondrogenesis (Supplementary Table S1). Wwp2 showed 6.24-fold change in mRNA expression.

Wwp2 is a downstream target of Sox 9 . The mRNA of Wwp2 is expressed in various adult mouse tissues, whereas Sox 9 mRNA is detected in specific tissues (Supplementary Fig. S1a,b). During limb bud development in mouse embryos, Wwp2 was expressed during mesenchymal condensations of limb buds at E12.5, and then in periarticular and proliferating chondrocytes at E16.5 (Fig. 2a). These expression patterns of Wwp2 during chondrogenesis coincided with that of Sox9. The expression of Wwp2 mRNA was completely abolished by the conditional inactivation of Sox9 using Prx1-Cremediated recombination in the limbs at E12.5 and Col2a1-Cremediated recombination in chondrocytes at E16.5 (Fig. 2a). Furthermore, adenoviral overexpression of Sox 9 in ATDC5 cells, a clonal murine chondrogenic cell line, induced the upregulation of Wwp2 significantly (Fig. 2b); in contrast, knockdown of Sox9 using short interfering RNA (siRNA) led to significant downregulation of its expression (Fig. 2b), and the expression of Wwp2 protein also showed a Sox9 dependency (Supplementary Fig. S1c,d). Thus, these results indicate that Wwp2 is a downstream target of Sox9, in vivo and in vitro.

Wwp2 transcription is regulated directly by Sox9. Next, we assessed whether Sox 9 regulates the transcription of Wwp2 directly. Putative Sox9-binding sites in the Wwp2 promoter region that are highly conserved among mammals (human, rat and mouse) were examined using in silico homology searches. A search of the region up to 4,000 nucleotides (nt) of Wwp2 exon 1 identified a putative Sox9-binding sequence (ACAAAGG), which lies between nt -529 and -523 and includes a nearly consensus motif for the Sox9-specific DNA-binding element ${ }^{22}$. To clarify the activity of the putative Sox9-responsive element of the $W w p 2$ promoter, a luciferase reporter assay was performed using a series of truncated fragments of the Wwp2 promoter cloned into a luciferase reporter vector (Fig. 2c). The results showed that co-transfection of $\mathrm{pW}_{-790}$ (which contains the $W w p 2$ promoter region from nt -790 to -64$)$ and a Sox9 expression vector into HEK293 cells induced an approximately twofold increase in the relative reporter activity compared with that observed for the control (Fig. 2c). In addition, this increase in reporter activity was mostly abolished by using the mutated $\mathrm{pW}_{-790}$ (termed $\mathrm{pWM}_{-790}$ ) or $\mathrm{pW}_{-391}$ (which lacked the putative 
a

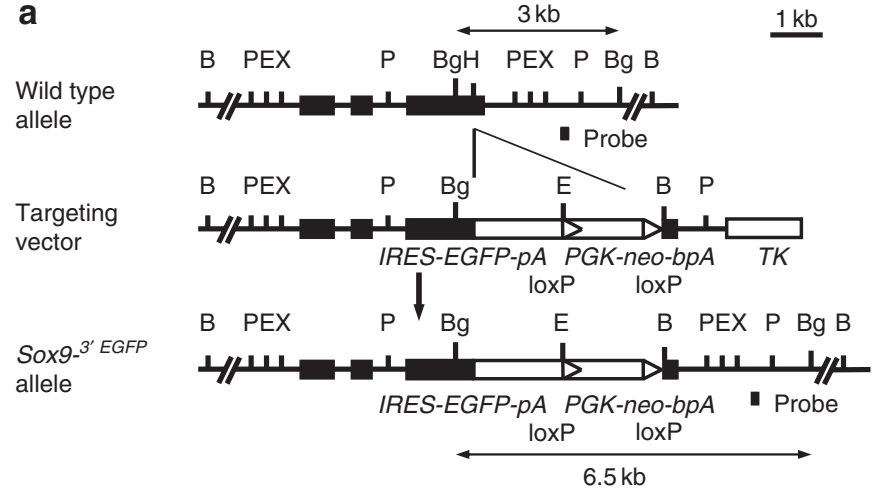

b

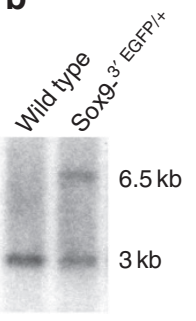

e

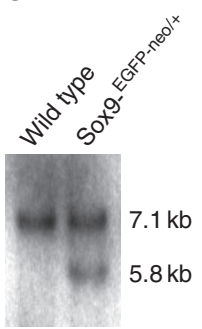

c
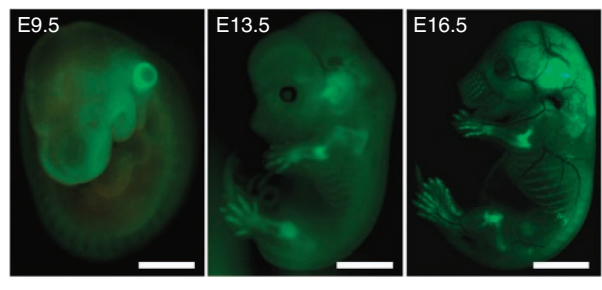

f

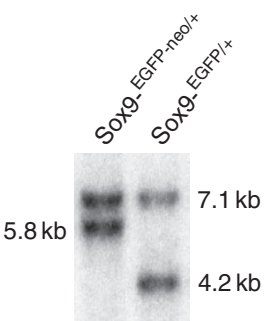

d

Wild type allele

Targeting
vector

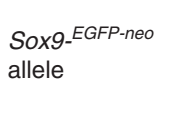

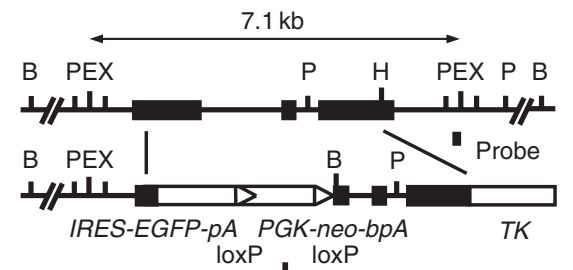

$B$ PEX IOxP

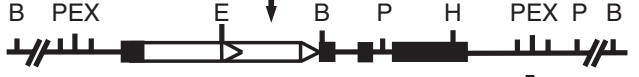

IRES-EGFP-pA PGK-neo-bpA loxP loxP

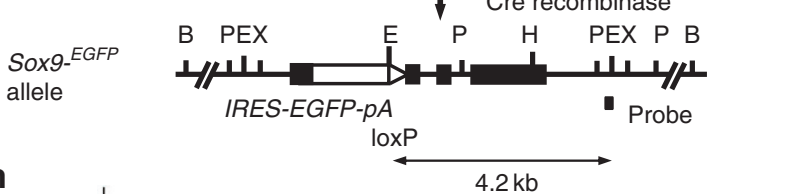

h

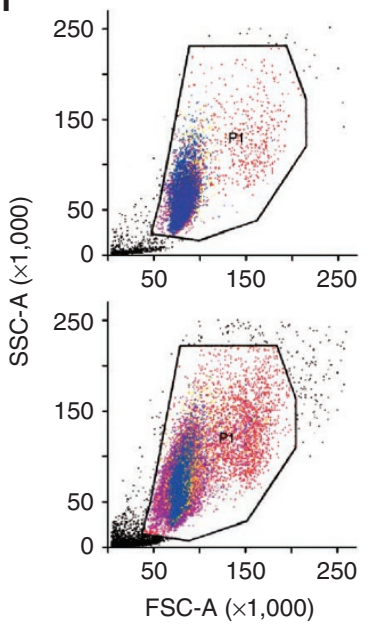
$\downarrow \quad$ Cre recombinase

$1 \mathrm{~kb}$

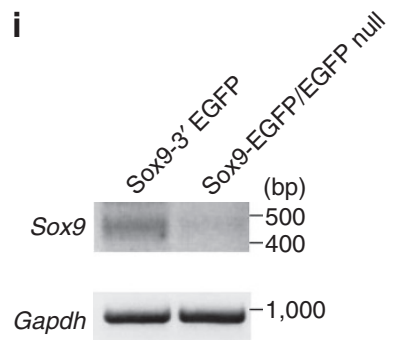

Figure 1 | Generation of Sox9-3' EGFP knock-in mice and Sox9-EGFP/EGFP null chimeras. (a) The structure of the genomic Sox9 locus, targeting vector and targeted allele for Sox9-3' EGFP knock-in mouse is indicated. Exons are depicted as closed boxes and intronic sequences are shown as solid lines. DNA fragments detected by Southern blot analysis are indicated by double arrows, with the restriction enzymes and the probe. The IRES-EGFP-pA/loxPflanked PGK-neo-bpA cassette is depicted as open boxes. B, BamHI; P, Pst1; E, EcoRl; X, Xbal; Bg, Bg/ll; H, Hpal. (b) Southern blot analysis of genomic DNA. Genomic DNA isolated from ES cell clones was digested with Bglll and was then hybridized with the 3' probe. The wild-type and mutant alleles were detected as $3 \mathrm{~kb}$ and $6.5 \mathrm{~kb}$ fragments, respectively. (c) EGFP expression in E9.5, E13.5 and E16.5 Sox9-3' EGFP knock-in embryos. EGFP expression was detectable in a Sox9-specific pattern during embryogenesis. Scale bars, $500 \mu \mathrm{m}$ (E9.5); $2 \mathrm{~mm}$ (E13.5) and $3 \mathrm{~mm}$ (E16.5). (d) The structure of the genomic Sox9 locus, targeting vector and targeted allele for Sox9-EGFP/EGFP null chimera is indicated. DNA fragments detected by Southern blot analysis are indicated by double arrows. (e) Southern blot analysis of genomic DNA. Genomic DNA isolated from ES cell clones was digested with EcoRI and was then hybridized with the $3^{\prime}$ probe. Wild-type and mutant alleles were detected as $7.1 \mathrm{~kb}$ and $5.8 \mathrm{~kb}$ fragments, respectively. (f) Southern blot analysis of the neodeleted Sox9 EGFP clones after Cre recombination. The neo-deleted Sox9 EGFP allele was detected as a $4.2 \mathrm{~kb}$ fragment. (g) Southern blot analysis of the neo-deleted Sox9 EGFP/EGFP-neo clones. (h) Dot plot cytograms show enrichment of EGFP-positive cells in Sox9-3' EGFP knock-in embryos (top panel) and Sox9-EGFP/EGFP null chimeras (bottom panel). Only cells from a P1 population showing high green fluorescence were sorted. (i) RT-PCR analysis of Sox9 expression using total RNA extracted from sorted EGFP-positive cells of Sox9-3' EGFP knock-in embryos and Sox9-EGFP/EGFP null chimeras.

Sox9-binding motif). To obtain direct evidence of Sox9-mediated binding to this site, a chromatin immunoprecipitation (ChIP) assay was performed. The ChIP experiment using adenoviruses expressing Sox9 in ATDC5 cells detected the specific binding of Sox9 to the endogenous $W w p 2$ promoter element (Fig. 2d). Moreover, an electrophoretic mobility shift assay (EMSA) provided further evidence that HA-tagged Sox9 bound to the DNA fragment of this site (Fig. 2e). Hence, we conclude that Sox 9 binds to the $W w p 2$ promoter element directly and transactivates $W w p 2$ expression.

$w w p 2$ knockdown induces palatal malformation in zebrafish. In zebrafish, the expression of $w w p 2$ in the cartilage of pharyngeal arches at 3 days post fertilization (dpf) was strongly reduced in embryos that were homozygous for $\operatorname{sox} 9 a$ or $s o x 9 b$ mutations (Supplementary Fig. S2a), whereas its expression in wild-type pharyngeal arches was detected at $2 \mathrm{dpf}$ (Supplementary Fig. S2b). Interestingly, morpholino-mediated knockdown of $w w p 2$ resulted in a small and fused palate, a phenotype that was reminiscent of sox9-mutant zebrafish (Fig. 2f ${ }^{23}$; however, the expression of sox9a and $s o x 9 b$, as detected in RT-PCR experiments, did not change after downregulation of $w w p 2$ expression (Supplementary Fig. S2c). Furthermore, the co-injection of both sox $9 a$ and sox $9 b$ mRNA together with $w w p 2$ morpholinos could partially rescue the palatal malformation (Fig. $2 \mathrm{~g}, \mathrm{~h}$ ). This restoration was thought to be due to the exogenous sox9-depedent induction of endogenous $w w p 2$ expression. These findings would be expected under the hypothesis 
a

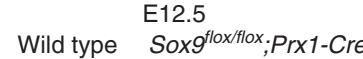
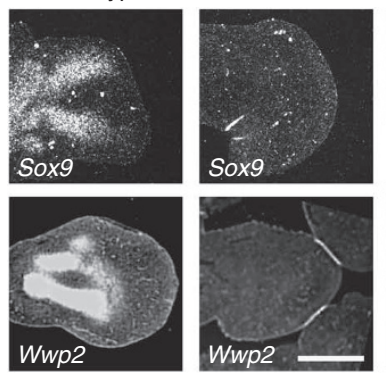

C

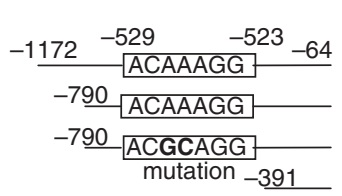

Wild type

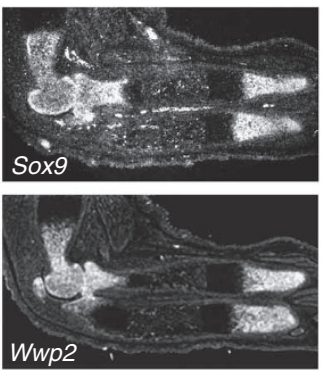

E16.5 Sox $9^{\text {floxflox }}$;Col2a1-Cre

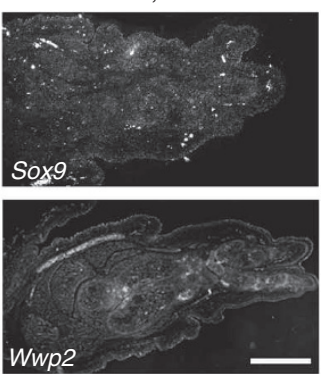

b
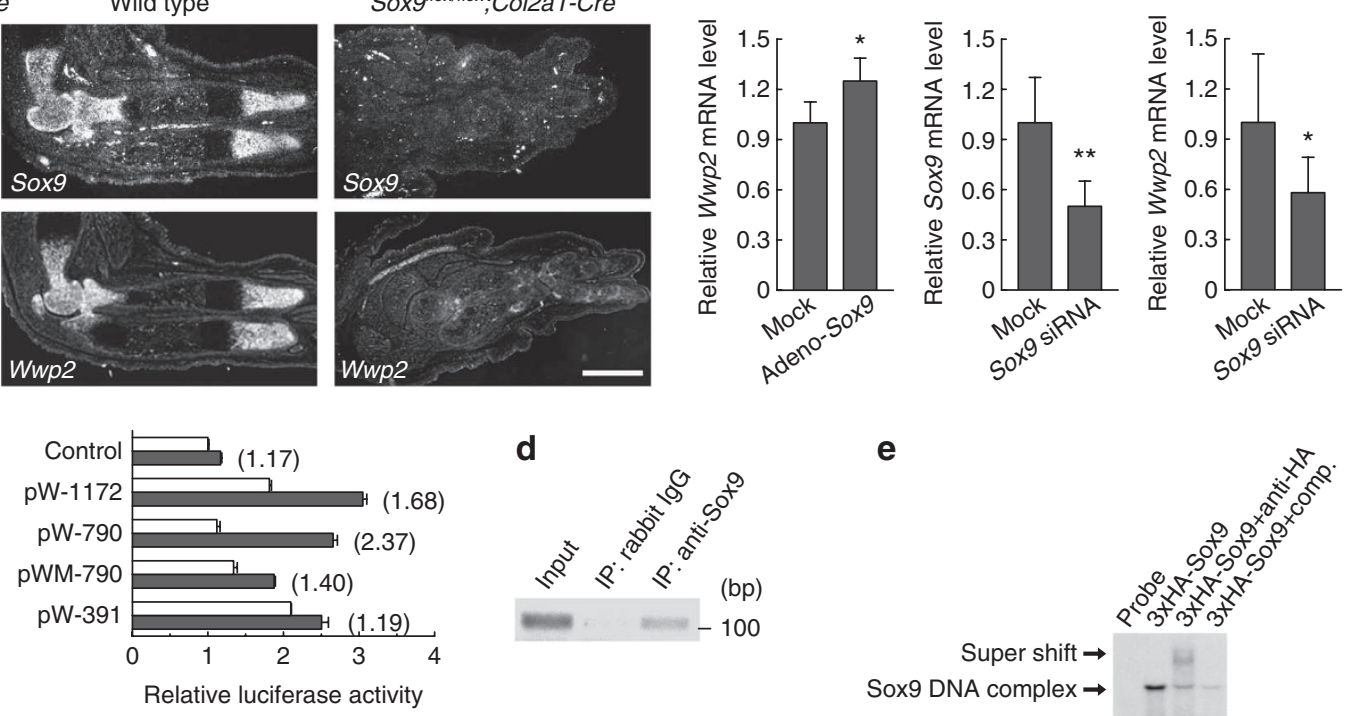

d

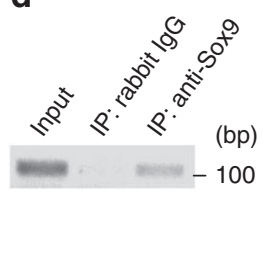

h

g
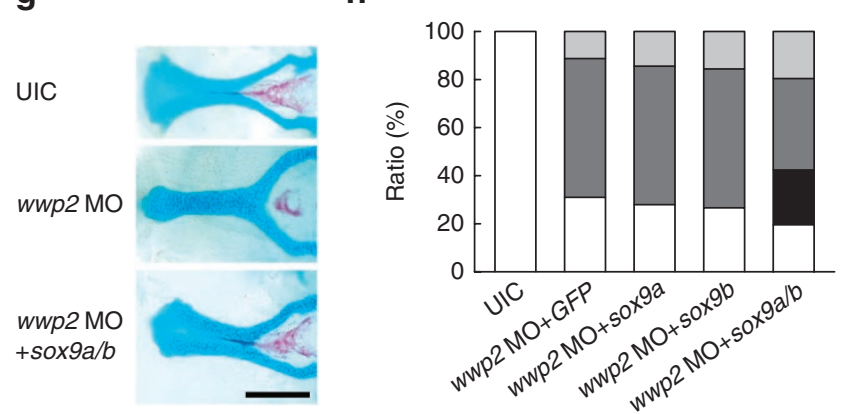

e

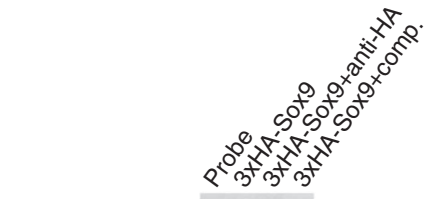

Super shift $\rightarrow$ Sox9 DNA complex $\rightarrow$

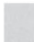

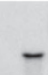

f
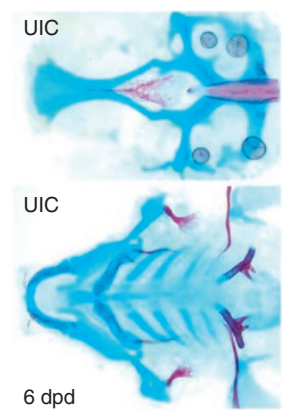

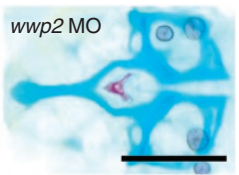

wwp2 MO

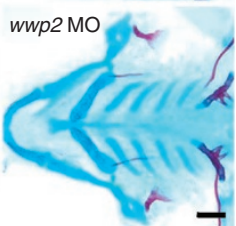

Figure 2 | Wwp2 is identified as a downstream target of Sox9 and regulates palatogenesis. (a) In situ hybridization of Sox9 and Wwp2 in limb buds

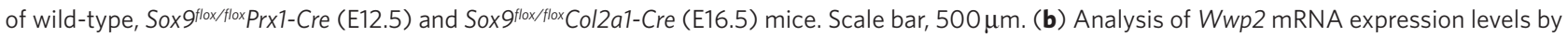
quantitative RT-PCR ( $q R T-P C R$ ) in ATDC5 cells. ATDC5 cells were infected with human Sox9-expressing adenovirus (left panel) or transfected with $150 \mathrm{nM}$ of siRNA for Sox9 (right panel; mean \pm s.d.; ${ }^{\star} P<0.05 ;{ }^{\star \star} P<0.01 ; n=5$ ). (c) Luciferase promoter analysis using truncated forms of the $W w p 2$ promoter in the presence or absence of the Sox 9 expression vector. HEK293 cells were transfected with the pW -1172 $(\mathrm{nt}-1172$ to -64$)$, the pW -790 (nt -790 to -64$)$, the $\mathrm{pW}_{-391}$ (nt -391 to -64 ) or the $\mathrm{pWM}_{-790}$ plasmid, which includes a 2 bp substitution mutation, in the presence (black bars) or absence (white bars) of Sox9 expression vector. The value in brackets represents the Sox9-dependent increase in ratio (mean \pm s.d.; $n=4)$. (d) The specific binding of Sox9 to the Wwp2 promoter element detected by ChIP assay using ATDC5 cells. DNA-protein complex was immunoprecipitated by anti-Sox9 antibody. (e) EMSA revealed the specific binding of Sox9 to the Wwp2 promoter element. comp., competitor (unlabelled probe). (f) Small and fused palate in zebrafish carrying morpholino-mediated knockdown of wwp2 at $6 \mathrm{dpf}$. Zebrafish embryos were stained with alcian blue and alizarin red. UIC, uninjected control; MO, morpholino-treated animals. Scale bar, $200 \mu \mathrm{m}$. (g, h) RNA rescue experiments for wwp2 knockdown in zebrafish using sox $9 a$ and/or sox $9 b$. (g) Palatal malformation induced by wwp2 knockdown was partially rescued by the co-injection of sox $9 a$ and sox $9 b$. Scale bar, $100 \mu \mathrm{m}$. (h) Single injection of sox $9 a$ or sox $9 b$ did not rescue the palatal malformation induced by wwp2 knockdown. UIC; $n=51, w w p 2 \mathrm{MO}+\mathrm{GFP} ; n=142$, wwp2 $\mathrm{MO}+\operatorname{sox} 9 a ; n=111$, wwp2 $\mathrm{MO}+$ sox9b; $n=109$, wwp2 $\mathrm{MO}+$ sox9a/b; $n=92$ (white, normal; black, partial rescue; dark grey, fused and/or small; light grey, death).

that Wwp2 has an essential role in chondrogenesis during palatogenesis, as an in vivo direct target of Sox9.

Wwp2 interacts physically with Sox9. The similar palatal phenotype observed in $s o x 9 a-$-, sox $9 b$ - and $w w p 2$-deficient zebrafish raises the possibility of physical and functional interactions between Sox9 and Wwp2. An IP experiment of endogenous Sox9 and myc-tagged Wwp2 using ATDC5 cells showed that Sox9 physically interacted with Wwp2 (Fig. 3a). Co-IP experiments using deletion mutations of Sox 9 (Fig. 3b) and Wwp2 (Fig. 3c) showed that the binding between Sox 9 and Wwp2 was mediated by the carboxy terminal transactivation domain of Sox9 (Fig. 3d) and by the amino terminus (N-terminus) of Wwp2 (Fig. 3e), and not by the WW domains, which have a direct role in the mediation of specific and distinct interactions with
Wwp2 substrates ${ }^{24}$. Indeed, Wwp2 did not stimulate ubiquitylationmediated Sox9 degradation (Supplementary Fig. S3).

Wwp2 promotes the transcriptional activity of Sox9. To elucidate whether Wwp2 is associated with Sox9 transcriptional activity, we investigated the nuclear localization of Wwp2 in chondrocytes, the nuclear translocation of Wwp2 in the presence or absence of exogenously expressed Sox9, and then performed luciferase reporter assays using a chondrocyte-specific p89/4×48 Col2a1 reporter and a Col11a2 reporter construct. Immunohistochemical staining exhibited the abundant nuclear localization of Sox9 and Wwp2 in proliferating and pre-hypertrophic chondrocytes of mouse limb buds at E16.5 (Fig. 3f). Interestingly, in vitro multiple immunofluorescence (IF) of $\mathrm{C} 3 \mathrm{H} 10 \mathrm{~T} 1 / 2$ cells, mouse pluripotent embryonic 
a

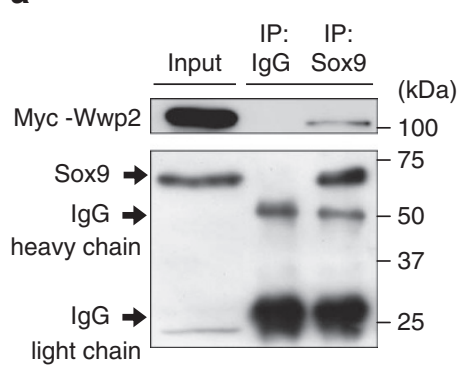

b

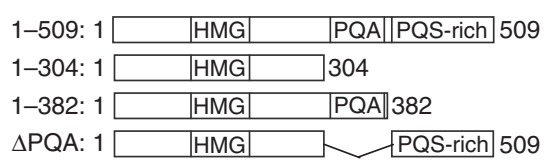

C

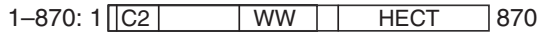

1-294: 1 पC2 $\square 294$

1-525: $1 \llbracket \mathrm{C} 2 \mathrm{C} 2 \mathrm{WW} \square 525$

$\triangle \mathrm{WW}: 1 \llbracket \mathrm{C} 2 \mathrm{HECT} 870$

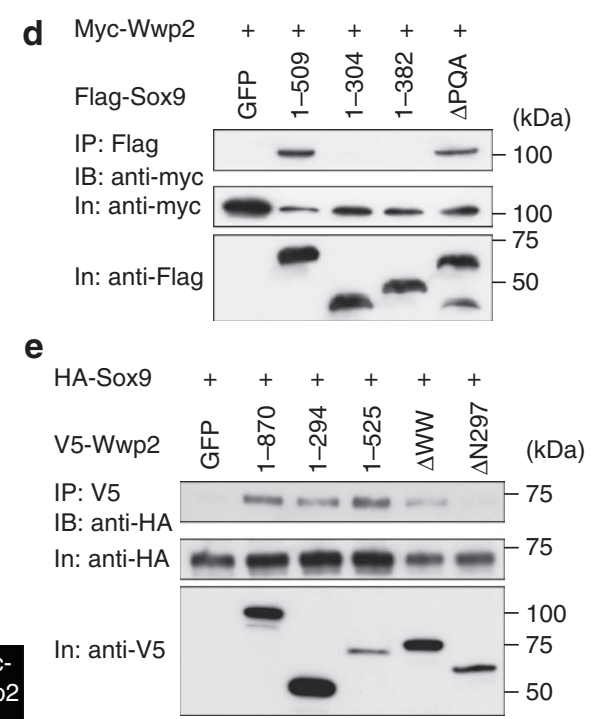

h

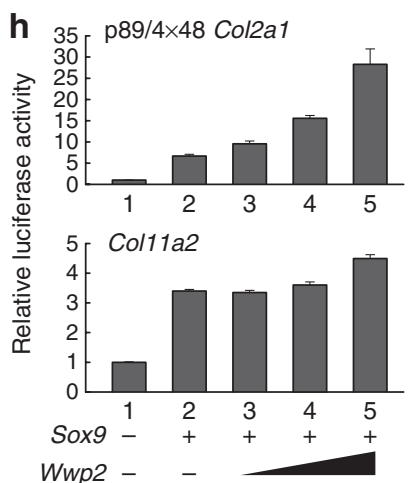

Figure $\mathbf{3}$ | Wwp2 activates Sox 9 by affecting its nuclear translocation. (a) Wwp2 interacted physically with endogenous Sox 9 as demonstrated by IP assay using ATDC5 cells. Myc-tagged Wwp2 was transfected into ATDC5 cells and total cell lysates were immunoprecipitated $48 \mathrm{~h}$ after transfection using rabbit IgG (negative control) or an anti-Sox9 antibody, followed by immunoblotting with an anti-myc antibody. (b) Schematic images of Sox9 deletion mutations. Sox9 contains a high-mobility group domain (HMG, residues 104-182); a proline, glutamine and alanine domain (PQA, residues 339-379); and a proline, glutamine and serine-rich domain (PQS-rich, residues 402-509). (c) Schematic images of Wwp2 deletion mutations. Wwp2 contains a calcium-dependent membrane targeting domain (C2, residues 19-115), four tryptophan-based WW domains (WW, residues 300-477) and a carboxy terminus domain that is homologous to that of E6-AP (HECT, residues 525-870). (d) Co-immunoprecipitation assays between myctagged Wwp2 and Flag-tagged deletion mutations of Sox9. GFP expression plasmid was used as a negative control. IB, immunoblotting; In, input. (e) Co-immunoprecipitation assays between HA-tagged Sox 9 and V5-tagged deletion mutations of Wwp2. GFP expression plasmid was used as a negative control. (f) Immunohistochemical analysis of Sox9 and Wwp2 in wild-type mouse limbs at E16.5 (DAB staining). Pz, proliferative zone; Pre$\mathrm{Hz}$, prehypertrophic zone; $\mathrm{Hz}$, hypertrophic zone. Scale bar, $100 \mu \mathrm{m}$. (g) Nuclear translocation of Wwp2 in the presence or absence of exogenously overexpressed Sox9 in C3H10T1/2 cells. The nuclear translocation of Wwp2 was associated with Sox9. Scale bar, 20 $\mu \mathrm{m}$. (h) Luciferase reporter assay using the p89/4×48 Col2a1 reporter plasmid or Col11a2 reporter plasmid in C3H10T1/2 cells. The reporter activities increased in a Wwp2 dose-dependent manner (mean \pm s.d.; $n=5$ ).

mesenchymal cell lines, co-transfected with HA-tagged Sox 9 and/or myc-tagged $W w p 2$ revealed that the exogenously expressed Sox 9 induced nuclear translocation of Wwp2 (Fig. 3g and Supplementary Fig. S4 for lower magnification image). This result indicates that Sox9 mediates the recruitment of Wwp2 from the cytosol into the nucleus in chondrocytes. Furthermore, Wwp2 co-transfected with Sox9 into C3H10T1/2 cells enhanced the Col2a1 and Col11a2 reporter activities in a dose-dependent manner (Fig. 3h). Thus, these results strongly suggest that Wwp2 promotes the transcriptional activity of Sox9 as a cofactor in the nucleus of chondrocytes.

Wwp2 binds to Med25 in chondrocytes. To identify the mechanism underlying the Wwp2-mediated promotion of Sox 9 transcriptional activity in chondrocytes, we sought other Wwp2-interacting proteins directly in chondrocytes using a yeast two-hybrid screening approach and identified Med25. Med25 exhibited strong physical interaction with Wwp2 in yeast (Fig. 4a). The mRNA of Med25 was expressed in various adult mouse tissues (Supplementary Fig. S5), and Med25 was detected in mouse limb bud chondrocytes at E16.5 (Fig. 4b). Med25 was reported as a specific target of the VP16 transcriptional activator ${ }^{25}$, and was shown to enhance retinoic acid receptor/retinoid X receptor-mediated transcription in co-operation with CBP and mediators ${ }^{26}$. In addition, in human disease, homozygous mutation of MED25 is responsible for Charcot-Marie-Tooth syndrome type $2 \mathrm{~B}^{27}$. However, the molecular functions of Med25 in chondrogenesis remain unknown.

med 25 knockdown induces palatal malformation in zebrafish. To clarify the role of Med25 in chondrogenesis, we investigated the in vivo functions of Med25 for skeletal formation in zebrafish. 


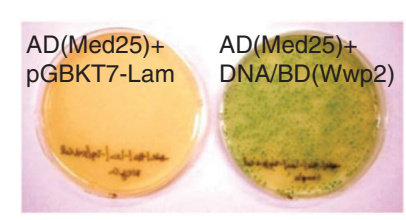

d
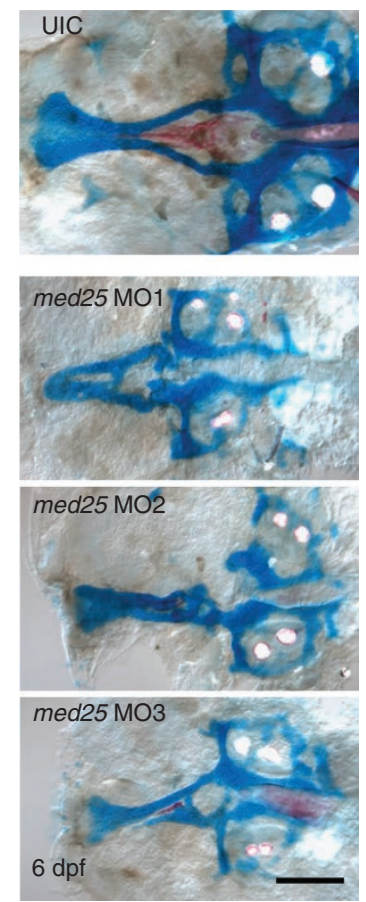

b
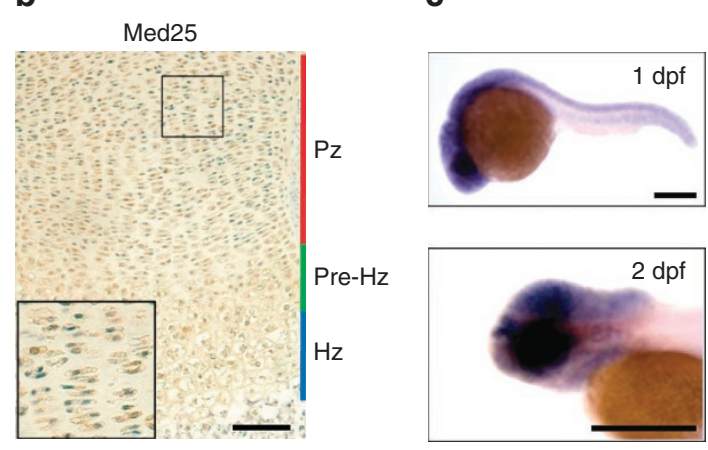

g

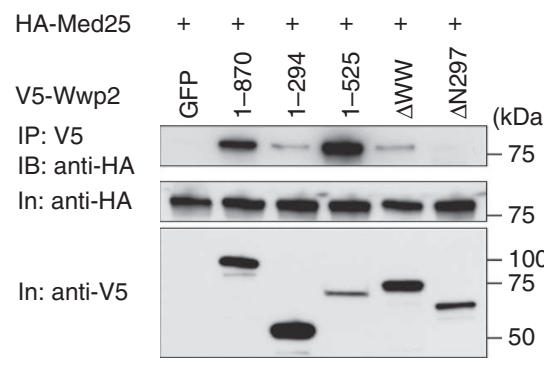

i

Flag-Sox9

HA-Med25

IP: HA

IB: anti-Flag

In: anti-Flag

In: anti-HA

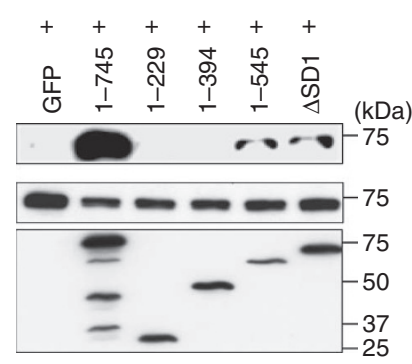

e

IP: IP:

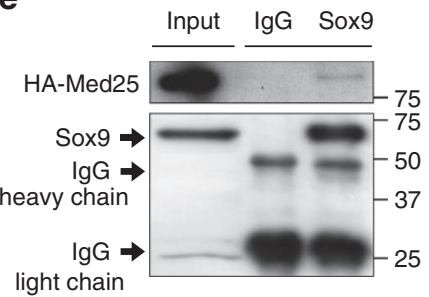

f

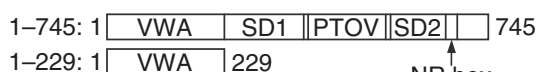

1-229: 1 VWA $229 \quad$ NR box

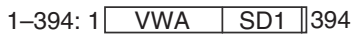

1-545: 1 \begin{tabular}{|l|l||l|}
\hline VWA & SD1 & PTOV 545
\end{tabular}

$\triangle S D 1: 1$ VWA $P$ PTOV $\|$ SD2 $\square 745$

h
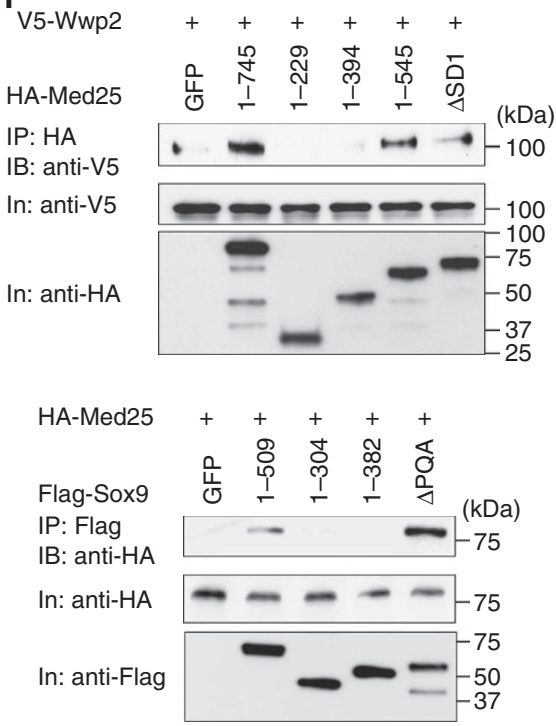

Figure 4 | Med25 is involved in palatogenesis and interacts physically with Sox 9 and $\mathbf{W} \mathbf{w p 2}$. (a) Verification of the physical interaction between Wwp2 and Med25 by a yeast two-hybrid assay. The AH109 yeast strain was co-transformed with the pGADT7 vector expressing full-length Med25 and the pGBKT7-Lam vector (negative control; left dish), or the pGBKT7 vector expressing full-length Wwp2 (right dish) was cultured on $\mathrm{SD} /-\mathrm{Ade} /-\mathrm{His} /$ - Leu/ - Trp/X- $\alpha$-Gal high-stringency plates. (b) Immunohistochemical analysis of Med25 in a wild-type mouse limb at E16.5 (DAB staining). Scale bar, $100 \mu \mathrm{m}$. (c) Whole-mount in situ hybridization of med25 in zebrafish at $1 \mathrm{dpf}$ (top panel) and $2 \mathrm{dpf}$ (bottom panel). Scale bar, $500 \mu \mathrm{m}$. (d) Morpholino-mediated knockdown of med25 in zebrafish induced palatal malformation. Three different kinds of palatal morphological malformations (med25 MOs1-3) were observed. Scale bar, $100 \mu \mathrm{m}$. (e) Med25 interacted physically with endogenous Sox 9 detected by IP assay using ATDC5 cells. HA-tagged Med 25 was transfected into ATDC 5 cells and a total cell lysate was immunoprecipitated $48 \mathrm{~h}$ after transfection using rabbit lgG (negative control) or an anti-Sox9 antibody, followed by immunoblotting with an anti-HA antibody. (f) Schematic images of Med25 deletion mutations. Med25 contains a von Willebrand factor type A domain (VWA, residues 1-228), a synapsin I domain 1 (SD1, residues 229-381), a conserved region found in prostate tumour overexpressed protein 1 domain (PTOV, residues 395-545), an SD2 domain (residues 554-731) and an NR box (residues 646-650). (g-j) Co-immunoprecipitation assays using deletion mutations of Sox9, Wwp2 or Med25. GFP expression plasmid was used as a negative control. (g) Immunoprecipitation between HA-tagged full-length Med25 and V5-tagged deletion mutations of Wwp2. (h) Immunoprecipitation between V5-tagged full-length Wwp2 and HA-tagged deletion mutations of Med25. (i) Immunoprecipitation between Flag-tagged full-length Sox9 and HA-tagged deletion mutations of Med25. (j) Immunoprecipitation between HA-tagged full-length Med25 and Flag-tagged deletion mutations of Sox9.

In situ hybridization showed that med25 was broadly expressed in the head, body and tail at 1 and $2 \mathrm{dpf}$ (Fig. 4c). Intriguingly, morpholino-mediated knockdown of med25 in zebrafish resulted in palatal malformation (Fig. 4d), which closely resembled the palatal phenotype of $w w p 2$ or $\operatorname{sox} 9$ mutants $^{23}$ (Fig. 2f). Furthermore, these palatal malformations could not be rescued by the co-injection of sox9a and sox $9 b$ mRNA or $w w p 2$ mRNA (Supplementary Fig. S6a,b). Also, the injection of med 25 mRNA with the wwp2 morpholinos had no influence on the palatal phenotype (Supplementary Fig. S6c). These findings suggest that Med25 transmits
Sox9 transcriptional activity to general transcription machinery in association with Wwp2 in palatogenesis.

Med25 interacts physically with Sox9 and Wwp2. The in vivo phenotypic similarities in palatogenesis between the knockdown of Sox9, Wwp2 and Med25, and the mutual interactions of Wwp2 with Sox 9 and Med25, strongly suggest a functional communication between Sox9 and Med25 during chondrogenesis. Therefore, to assess whether Sox9 interacts physically and/or functionally with Med25, an IP assay was performed using ATDC5 cells transfected 


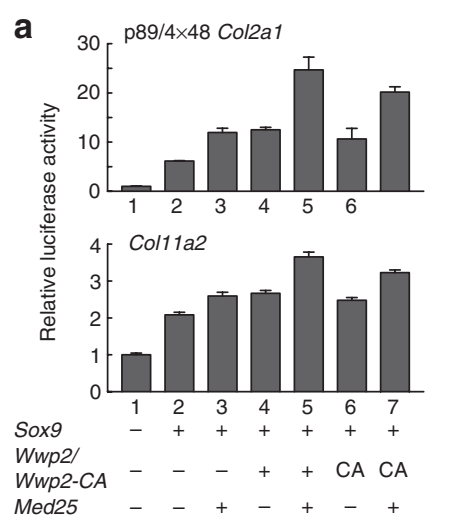

e

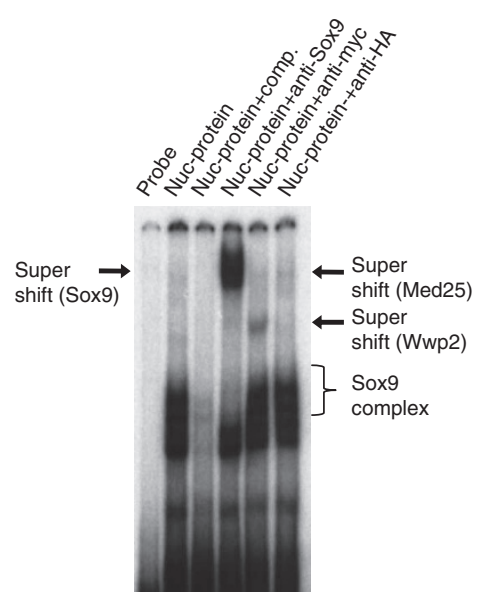

b
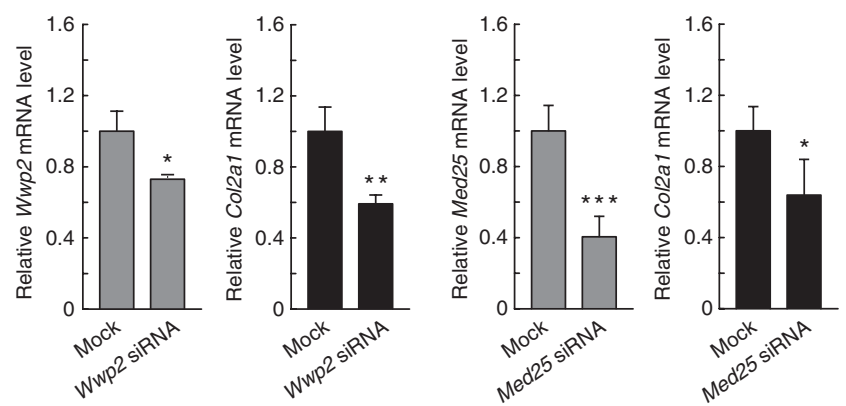

f

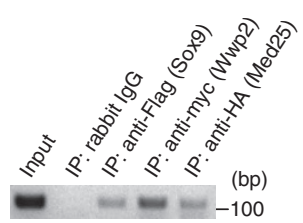

g

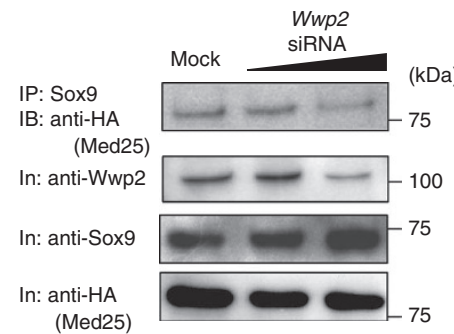

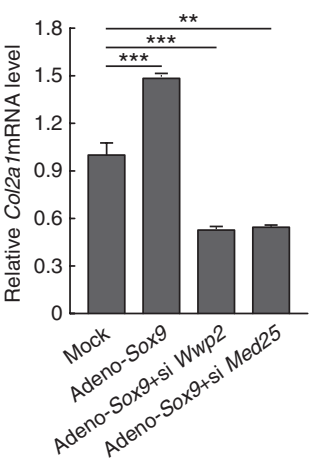

d

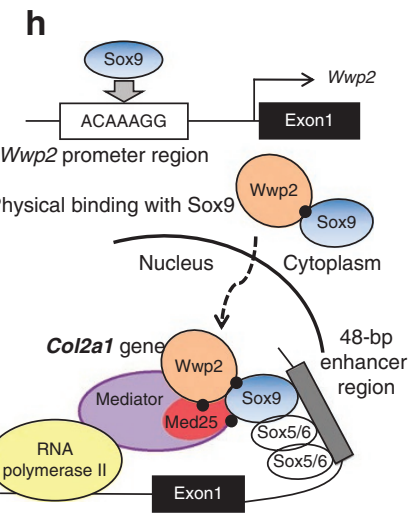

Figure 5 | Wwp2 mediates formation of transcriptional complex with Sox 9 and Med25 and regulates Sox 9 transcriptional activity. (a) Luciferase reporter assay using the p89/4×48 Col2a1 reporter and Col11a2 reporter plasmid in C3H10T1/2 cells. Wwp2-CA includes a mutation in the HECT domain of Wwp2. (mean \pm s.d.; $n=5$ ). (b, c) Knockdown of Wwp2 (b) or Med25 (c) using siRNAs led to the downregulation of Col2a1 transcription in C3H10T1/2 cells detected by qRT-PCR. (mean \pm s.d.; $\left.{ }^{\star} P<0.05,{ }^{* \star} P<0.01,{ }^{* \star *} P<0.001 ; n=4\right)$. (d) Downregulation of Col2a1 induced by knockdown of Wwp2 or Med25 in C3H10T1/2 cells was not restored by exogenous Sox9 overexpression. (mean \pm s.d.; Dunnett's test, ${ }^{\star \star} P<0.01 ;{ }^{\star \star \star} P<0.001 ; n=5$ ). (e) EMSA using nuclear protein from $\mathrm{C} 3 \mathrm{H} 10 \mathrm{~T} 1 / 2$ cells that were co-transfected with Flag-tagged Sox9, myc-tagged Wwp2 and HA-tagged Med25. The 48-bp Col2a1 enhancer oligonucleotide probe was used. Nuc-protein, nuclear protein. (f) ChIP assay detected recruitment of Sox9, Wwp2 and Med25 to the chondrocyte-specific enhancer region of the Col2a1 gene. Cell lysate extracted from C3H10T1/2 cells that were co-transfected with Flag-tagged Sox9, myc-tagged Wwp2 and HA-tagged Med25 was immunoprecipitated with antibodies for each tag. (g) Downregulation of Wwp2 led to the attenuation of the physical binding between Sox 9 and Med25. The Wwp2 siRNA was initially transfected into ATDC5 cells and HA-tagged Med25 was additionally transfected $24 \mathrm{~h}$ after transfection with Wwp2 siRNA. Nuclear protein was immunoprecipitated with an anti-Sox 9 antibody, followed by immunoblotting with an anti-HA antibody. (h) Model describing the mechanism underlying the physical and functional interaction between Sox9, Wwp2 and Med25. Sox9 regulates the transcription of Wwp2 and mediates the nuclear translocation of Wwp2, resulting in the formation of a transcriptional complex, including RNA polymerase II and Sox 5/6, which regulates the expression of the Col2a1 gene.

with HA-tagged Med25. The result showed that Med25 interacted physically with endogenous Sox9 (Fig. 4e). Co-IP experiments using deletion mutations of Sox9, Wwp2 and Med25 (Figs 3b,c and 4f) led to the following results. First, Med25 interacted physically with the N-terminus of Wwp2 and Wwp2 bound to the PTOV domain of Med25, which includes its transactivation domain ${ }^{26}$ (Fig. 4g,h), and not to the SD1 domain of Med25, which contains the putative PY motif (the WW domains of Wwp2 have a preference for the PY motif in the binding of substrate proteins) ${ }^{28,29}$ Indeed, Wwp2 did not stimulate ubiquitylation-mediated Med25 degradation (Supplementary Fig. S7). Second, Med25 and Sox9 interacted with each transactivation domain (Fig. 4i,j). Thus, these physical interactions between Sox9, Wwp2 and Med25 suggest their functional interactions. In addition, multiple IF experiments revealed that Med25 was expressed in cell nucleus, and the nuclear translocation of Wwp2 depended on Sox9 and not on Med25 (Supplementary Fig. S8a,b).
Wwp2 and Med25 augment Sox9 transcriptional activity. Luciferase reporter assays using the $\mathrm{p} 89 / 4 \times 48$ Col2al reporter or the Col11a2 reporter showed that co-transfection with Sox9, Wwp2 and $M e d 25$ enhanced reporter activity significantly compared with that observed for Sox 9 and Wwp2 or for Sox 9 and Med25 (Fig. 5a). Interestingly, Wwp2 carrying a mutation in the HECT domain, which leads to loss of its catalytic function as an E3 ligase ( $W w p 2-$ $C A$; change of the cysteine residue at position 838 to an alanine), was still capable of increasing Sox9-dependent Col2a1 and Col11a2 luciferase activity (Fig. 5a). However, the slightly lower luciferase activity observed when using $W w p 2-C A$ would indicate a loss of ubiquitylation-dependent activation by other molecules that regulate the transcription of Col2a1 and Col11a2. Knockdown of either Wwp2 or Med25 in C3H10T1/2 cells resulted in significant downregulation of Col2al transcripts (Fig. 5b,c). In addition, this in vitro downregulation was not restored by adenoviral overexpression of 
Sox9 (Fig. 5d), which strongly suggests that Wwp2 and Med25 are key mediators of Sox9-dependent transcription.

Wwp2 forms a transcriptional complex with Sox9 and Med25. To obtain direct evidence of the formation of transcriptional complex through binding of these proteins to the chondrocytespecific enhancer of the Col2al gene, we performed EMSA and ChIP assays using C3H10T1/2 cells, and revealed that Sox9, Wwp2 and Med25 were recruited onto the Col2al enhancer as a transcriptional complex (Fig. 5e,f). Importantly, knockdown of Wwp2 in ATDC5 cells attenuated the physical interaction between endogenous Sox9 and HA-tagged Med25 (Fig. 5g). Thus, these results indicate that Wwp2 mediates the interaction between Sox 9 and Med25 via mutual physical binding and regulates Sox9 transcriptional activity.

\section{Discussion}

Here we identified Wwp2 as a Sox9 downstream target using FACS/ microarray gene expression analysis and confirm the findings by in situ hybridization in conditional Sox9 knockout embryos and overexpression and knockdown of Sox9 in in vitro cells. Furthermore, both EMSA and ChIP assays using an anti-Sox9 antibody and reporter assays with luciferase reporter constructs indicate that Sox 9 binds to the $W w p 2$ promoter and regulates $W w p 2$ expression directly. Zou et al. ${ }^{30}$ recently reported that Wwp2 is detected in cartilaginous regions of the skull that are also positive for Sox9 and that Sox 9 binds to an intronic region of Wwp2, which mediates the Sox9dependent expression of $W w p 2$. Thus, these findings indicate that Sox9 regulates the cartilaginous expression of $W w p 2$ directly.

Palatogenesis is controlled by multiple genetic factors, including genes encoding extracellular matrix proteins, growth factors and cell adhesion molecules. Alternations in the synthesis and accumulation of extracellular matrix proteins, including type II, IX, XI collagens, and proteoglycan ${ }^{10-12}$ impair palatogenesis, all of that are regulated by Sox $9^{1,7-9}$. Indeed, mutations in or around SOX9 gene result in palatal malformations in human and a mouse model. Thus, it is obvious that Sox9 has a crucial role in palatogenesis. In this study, we show that Wwp2 is required for palatogenesis, mediated by formation of a transcriptional complex with Sox 9 and Med25. Morpholino-mediated knockdown of $w w p 2$ in zebrafish results in palatal malformation, which is comparable to that in Wwp2deficient mice reported by Zou et al..$^{30}$ They also show that Wwp2 interacts with Goosecoid to facilitate its monoubiquitylation and augments its transcriptional activity for Sox6 expression ${ }^{30}$. Taking together with the previous findings that Sox6 cooperates with Sox9 to directly activate downstream target genes encoding extracellular matrix proteins ${ }^{7,8}$, it is likely that Wwp2 is essential for palatogenesis through Sox9 transcriptional activity (as shown in Fig. 5h).

Because Sox9 is required for the development of several tissues, which are characterized by very different genetic programs, other transcription factors and coactivators should provide specificity to the function of Sox9 in each of these cell types. Transcription factors form a large complex including the Mediator complex that conveys regulatory information from enhancer elements to the basal transcription machinery and acts as a bridge between transcription factors and RNA polymerase II. A recent study using human hepatocytes shows that Med25 is required for the recruitment of RNA polymerase II to the promoter of hepatocyte nuclear factor $4 \alpha$ and interacts with hepatocyte nuclear factor $4 \alpha$ through its transactivation domain ${ }^{31}$. Hence, it is likely that Med25 recruits RNA polymerase II on the promoter of Sox 9 target genes. Med25 is believed to function in chromatin modification and pre-initiation complex assembly by recruiting CBP and the Mediator complex ${ }^{26}$. In addition, Trap230/Med12 is known as a coactivator of Sox9dependent transcriptional regulation ${ }^{14}$. The phenotypic similarity in palatogenesis among sox $9 a /$ sox $9 b, w w p 2$, med 25 and Trap230/ med12 mutants ${ }^{14,23}$ and the in vitro physical interaction between
Sox9/Trap230, Sox9/Med25, Sox9/Wwp2 and Wwp2/Med25 may reflect a possible regulatory axis, which would include a Sox9Wwp2-Med25-Trap complex.

In conclusion, Sox 9 stimulates $W w p 2$ expression and induces the translocation of Wwp2 into the nucleus of chondrocytes. Wwp2 mediates the binding between Sox 9 and Med25, resulting in transactivation of target genes (Fig. 5h). This molecular mechanism is thought to be involved in palatogenesis in vivo. Our findings provide new insights into the regulatory mechanisms of Sox9 transcriptional activity during chondrogenesis and suggest new ideas regarding the molecular basis of the pathogenesis of cleft palate.

\section{Methods}

Mice. Sox9-3' EGFP knock-in mice were generated using the targeting vector that spanned a $7.7 \mathrm{~kb}$ fragment of the mouse $129 \mathrm{SvEv}$ Sox9 gene ${ }^{32}$. An IRES-EGFP$p A /$ loxP-flanked PGK-neo-bpA cassette was inserted into an HpaI site within the $3^{\prime}$-untranslated region in exon 3 . The targeting vector was introduced into PC3 mouse ES cells. Mouse chimeras were generated by injection of mutant ES cell clones into C57BL/6 host blastocysts; the resulting chimeras were bred with C57BL/6 mice to generate Sox9-3' EGFP knock-in heterozygous mice.

Sox9-EGFP/EGFP null chimeras were generated using a $4.5 \mathrm{~kb}$ NotI-SacII $5^{\prime}$ fragment and a $2.5 \mathrm{~kb}$ SacI-SacI $3^{\prime}$ fragment derived from the Sox 9 locus ${ }^{33}$. An IRES-EGFP-pA/loxP-flanked PGK-neo- $b p A$ cassette was introduced into the first exon of Sox 9 , simultaneously deleting the translation start site and a portion of the high-mobility group DNA-binding domain. The targeting vector was introduced into AB-1 ES cells. To reuse G418 selection, the loxP-flanked neo cassette was excised from the Sox9 EGFP-neo/ + ES cell lines by transient expression of Cre recombinase. Neo-deleted Sox 9 EGFP/ + ES cell clones were identified by Southern blot and verified by G418 selection. The Sox9 EGFP/ + ES cell lines were then retargeted with the original vector, as described above, and Sox9-EGFP/EGFP null ES cells were verified by Southern blot. The Sox9-EGFP/EGFP null ES cells were microinjected into C57BL/6 mouse blastocysts to generate chimeric embryos. Experimental procedures and animal maintenance followed institutional guidelines and were approved by local authorities.

FACS and microarray analysis. The limb buds of E12.5 Sox9-3' EGFP knock-in and Sox9-EGFP/EGFP null chimeric embryos were dissected and dissociated into single cells using collagenase and disperse digestion. EGFP-positive cells were sorted using a FACSAria flow cytometer (Becton-Dickinson). Deletion of Sox9 in the cells harvested from Sox9-EGFP/EGFP null chimeric embryos was verified by PCR (Supplementary Table S2). Total RNA was extracted and amplified according to the instructions provided by Affymetrix. Microarray analysis using the Affymetrix Mouse Genome 430 2.0 Array was performed according to the manufacturer's instructions.

In situ hybridization of mouse embryos. The development-associated expression patterns of Sox 9 and $W w p 2$ mRNA in the limb buds of wild-type, Sox $9^{\text {flox/flox}}$ Prx 1 Cre and Sox $9^{f l o x / f l o x}$ Col2a1-Cre embryos were analysed using the hybridization probes for Sox9 (255bp) and Wwp2 (440 bp).

Yeast two-hybrid screen. A yeast two-hybrid assay was carried out using Matchmaker Library Construction \& Screening Kits (Clontech), according to the manufacturer's instructions. The full-length mouse $W w p 2 \mathrm{cDNA}$ inserted into pGBKT7 was used as the bait vector. Med25 identified using a cDNA library from mouse rib chondrocytes was inserted into pGADT7 (prey vector). The pGADT7 vector expressing full-length Med25 was retransformed into the AH109 yeast strain, together with the pGBKT7 vector expressing Wwp2 or with the pGBKT7Lam vector (negative control), and cultured on SD/ - Ade/ - His/ - Leu/ - Trp/ $\mathrm{X}-\alpha$-Gal high-stringency plates.

Zebrafish. Developing and adult zebrafish were maintained using standard methods. Wild-type matings were obtained from the Oregon AB line. To suppress pigmentation, embryos were raised in zebrafish aquatic system water containing 1-phenyl-2-thiourea (0.003\%) (Sigma). Zebrafish care was in accordance with the guidelines of the Children's Hospital Boston, University of Oregon and Murayama Medical Center.

In situ hybridization of zebrafish. Whole-mount in situ hybridization was carried out in zebrafish embryos at 1 and 2 dpf. Digoxigenin-labelled RNA probes were generated using the T7 and T3 labelling kit (Roche), according to the manufacturer's instructions. Signals were detected with an alkaline phosphatase-conjugated anti-digoxigenin antibody (Roche) and 4-nitro blue tetrazolium and 5-bromo-4chloro-3-indolyl-phosphate. The primer sequences of the probes were described in Supplementary Table S2.

Morpholino knockdown and RNA rescue in zebrafish. Gene Tools (Philomath; http://www.gene-tools.com) supplied morpholino oligonucleotides (MOs) with the 
sequences: zebrafish $w w p 2$ splice blocker-MO, $5^{\prime}$-CAGTGTGAATGCAGTGTTTT ACCCA-3' and zebrafish med 25 ATG blocker, 5'-TATGGGTCACCTTTGGTCT ACAGCC-3'. We injected one- or two-cell stage zebrafish embryos with $\sim 1-2 \mathrm{nl}$ of morpholinos (20-25 ng for wwp2 splice blocker-MO and 5-10 ng for med 25 ATG blocker). In RNA rescue experiments, we performed a series of experiments injecting each morpholino with either $50-500 \mathrm{pg}$ of sox $9 a$ and/or sox $9 b, w w p 2$ or med 25 mRNA.

Zebrafish cartilage staining. Zebrafish embryos at 4-7 dpf were stained with alcian blue and alizarin red and were flat mounted ${ }^{34}$. Images of stained, flat-mounted cartilages were captured using a Leica DMLB upright microscope and Spot camera (Diagnostic Instruments, Inc).

siRNA. The mouse Sox9-specific siRNA (MISSION siRNA) was purchased from Sigma-Aldrich and the mouse Wwp2- and Med25-specific siRNAs (ON-TARGET plus SMARTpool) were purchased from Thermo Scientific Dharmacon. For mRNA expression analysis, each siRNA construct was transfected individually into ATDC 5 or C3H10T1/2 cells (final concentration $150 \mathrm{nM}$ ) according to the manufacturer's protocol. Total RNA was extracted $48 \mathrm{~h}$ after transfection. A total of $1 \mu \mathrm{g}$ of total RNA was used to synthesize cDNA using the Transcriptor First-Strand cDNA Synthesis Kit (Roche). Quantitative RT-PCR was performed using a LightCycler Instrument and a LightCycler FastStart DNA Master SYBR Green 1 Kit (Roche). The primer sequences for quantitative RT-PCR were described in Supplementary Table S2.

Luciferase assays. In promoter analysis, mouse $W w p 2$ promoter fragments located upstream of the $5^{\prime}$-flanking region of exon $1(-1,172 \mathrm{bp}$ to $-64 \mathrm{bp},-790 \mathrm{bp}$ to $-64 \mathrm{bp}$, and $-391 \mathrm{bp}$ to $-64 \mathrm{bp}$ ) were subcloned into the pGL3-basic vector (Promega) to generate the Wwp2 promoter/luciferase reporter constructs, designated $\mathrm{pW}_{-1172}, \mathrm{pW}_{-790}$ and $\mathrm{pW}_{-391}$, respectively. The mutated $\mathrm{pW}_{-790}\left(\right.$ termed $\mathrm{pWM}_{-790}$ ) construct, which includes a $2 \mathrm{bp}$ substitution mutation in the nearly consensus binding site for high-mobility group box proteins, was produced using the QuickChange site-directed mutagenesis kit (Stratagene), according to the manufacturer's protocol. In Col2a1 or Col1 1a2 reporter analysis, a chondrocyte-specific p89/4×48 Col2a1 reporter or a Col11a2-luc reporter construct was used. pcDNA-renilla luciferase was used as an internal control. The plasmids were transfected using the FuGene HD transfection reagent (Roche) into HEK293 or $\mathrm{C} 3 \mathrm{H} 10 \mathrm{~T} 1 / 2$ cells. Luciferase activity was assayed $48 \mathrm{~h}$ after transfection, accord ing to the manufacturer's instructions.

ChIP. ChIP assays were performed using the ChIP Kit (Abcam), according to the manufacturer's protocol. ATDC5 cells cultured in six-well plates were infected with adenoviruses expressing Sox $9 ; 48 \mathrm{~h}$ after infection, the fragments of cross-linked chromatin were immunoprecipitated with $3 \mu \mathrm{g}$ of rabbit IgG (Cell signaling, $1 \mathrm{mg} \mathrm{ml}^{-1}$ ) or anti-Sox9 antibody (Chemicon, $1 \mathrm{mg} \mathrm{ml}^{-1}$ ). PCR primers were designed to contain a putative binding site within the mouse $W w p 2$ promoter region (Supplementary Table S2). C3H10T1/2 cells were co-transfected with Flag-tagged Sox9, 6× myc-tagged Wwp2 and 3× HA-tagged Med25 expression vectors, and ChIP assay was performed according to the same scheme using $3 \mu \mathrm{g}$ of anti-Flag (Abcam, $1 \mathrm{mg} \mathrm{ml}^{-1}$ ), anti-myc (Abcam, $1 \mathrm{mg} \mathrm{ml}^{-1}$ ) and anti-HA (Santa Cruz, $1 \mathrm{mg} \mathrm{ml}^{-1}$ ) antibodies. PCR was performed to detect the co-precipitation of the Col2al enhancer region (Supplementary Table S2).

EMSA. A DNA probe corresponding to the putative Sox 9 binding sequence of the $5^{\prime}$-flanking region of the $W w p 2$ gene was prepared by annealing complementary oligonucleotides (Supplementary Table S2). The Sox9 protein was synthesized in vitro using a transcription/translation system (Promega). DNA-binding reactions and EMSA with the $48 \mathrm{bp}$ Col2al enhancer probe were performed using nuclear proteins of $\mathrm{C} 3 \mathrm{H} 10 \mathrm{~T} 1 / 2$ cells co-transfected with $6 \times$ myc-tagged Wwp2 and $3 \times$ HA-tagged Med25 expression vectors ${ }^{35}$ (Supplementary Table S2).

Immunoprecipitation. Tagged plasmids were transfected into ATDC5, C3H10T1/2 or HEK293 cells, and after $48 \mathrm{~h}$, cells were lysed in buffer $(50 \mathrm{mM}$ Tris, $150 \mathrm{mM} \mathrm{NaCl}, 1 \mathrm{mM}$ EDTA and $1 \%$ Triton X-100). Protein complexes were immunoprecipitated using protein $\mathrm{G}$ agarose with $3 \mu \mathrm{g}$ of anti-Sox 9 antibody (Chemicon, $1 \mathrm{mg} \mathrm{ml}^{-1}$ ), $3 \mu \mathrm{g}$ of anti-V5 antibody (MBL, $1 \mathrm{mg} \mathrm{ml}^{-1}$ ), $30 \mu \mathrm{l}$ of Ezview Red ANTI-FLAG M2 Affinity Gel (Sigma) or $30 \mu$ l of Anti-HA Affinity Matrix (Roche). Immunoprecipitated proteins were analysed by immunoblotting using each antibody.

Immunohistochemical analysis. Mouse limb buds at E16.5 were fixed in $4 \%$ paraformaldehyde and embedded in paraffin. Immunohistochemical analysis was performed using 3,3'-diaminobenzidine (DAB) staining (Zymed), and hematoxylin was used as a counterstain. The following primary antibodies were used: goat polyclonal anti-Sox9 antibody (Santa Cruz, $0.2 \mathrm{mg} \mathrm{ml}^{-1}$ ), rabbit polyclonal anti-AIP2 antibody (Santa Cruz, $0.2 \mathrm{mg} \mathrm{ml}^{-1}$ ) and goat polyclonal anti-Med25 antibody (Santa Cruz, $0.2 \mathrm{mg} \mathrm{ml}^{-1}$ ) diluted in antibody diluent (Zymed) to 1:100.

IF staining. IF staining was performed using $\mathrm{C} 3 \mathrm{H} 10 \mathrm{~T} 1 / 2$ cells. Cells were fixed and permeabilized with cold methanol for $25 \mathrm{~min}$ at $4^{\circ} \mathrm{C}$. After washing, cells were blocked with a fresh blocking solution (4\% donkey serum in PBS containing 0.05\% Tween-20 (PBST)) for $30 \mathrm{~min}$ and were incubated with a rabbit anti-HA antibody (Santa Cruz, $0.2 \mathrm{mg} \mathrm{ml}^{-1}$ ) diluted to 1:200 in 1\% donkey serum/PBS containing $0.05 \%$ Tween-20 for $1 \mathrm{~h}$ at room temperature. Cells were washed and incubated with an fluorescein isothiocyanate-conjugated donkey anti-rabbit IgG antibody (Jackson ImmunoResearch Laboratories, $0.5 \mathrm{mg} \mathrm{ml}^{-1}$ ), diluted to 1:1,000 for $1 \mathrm{~h}$ at room temperature. After washing, the procedure used for multiple staining was repeated using a goat anti-myc antibody (Abcam, $1 \mathrm{mg} \mathrm{ml}^{-1}$ ) or a goat anti-Flag antibody (Abcam, $1 \mathrm{mg} \mathrm{ml}^{-1} ; 1: 200$ ), and a TRITC-conjugated donkey anti-goat IgG antibody (1:1,000; Jackson ImmunoResearch Laboratories, $\left.0.5 \mathrm{mg} \mathrm{ml}^{-1}\right)$. Confocal images were obtained with a confocal laser microscope (Nikon Instruments Inc.).

Statistical analysis. Statistical evaluation was conducted using the Student's $t$-test or Dunnett's test. All error bars represent s.d. and significance was assessed as $P<0.05$.

\section{References}

1. Akiyama, H., Chaboissier, M. C., Martin, J. F., Schedl, A. \& de Crombrugghe, B. The transcription factor Sox 9 has essential roles in successive steps of the chondrocyte differentiation pathway and is required for expression of Sox 5 and Sox6. Genes. Dev. 16, 2813-2828 (2002)

2. Akiyama, H. et al. Interactions between Sox 9 and beta-catenin control chondrocyte differentiation. Genes. Dev. 18, 1072-1087 (2004).

3. Wagner, T. et al. Autosomal sex reversal and campomelic dysplasia are caused by mutations in and around the SRY-related gene SOX9. Cell 79, 1111-1120 (1994).

4. Foster, J. W. et al. Campomelic dysplasia and autosomal sex reversal caused by mutations in an SRY-related gene. Nature 372, 525-530 (1994).

5. Benko, S. et al. Highly conserved non-coding elements on either side of SOX9 associated with Pierre Robin sequence. Nat. Genet. 41, 359-364 (2009).

6. Bi, W. et al. Haploinsufficiency of Sox9 results in defective cartilage primordia and premature skeletal mineralization. Proc. Natl Acad. Sci. USA 98, 6698-6703 (2001).

7. Smits, P. et al. The transcription factors L-Sox 5 and Sox 6 are essential for cartilage formation. Dev. Cell. 1, 277-290 (2001).

8. Lefebvre, V., Li, P. \& de Crombrugghe, B. A new long form of Sox 5 (L-Sox5), Sox6 and Sox 9 are coexpressed in chondrogenesis and cooperatively activate the type II collagen gene. EMBO J. 17, 5718-5733 (1998).

9. Han, Y. \& Lefebvre, V. L-Sox 5 and Sox 6 drive expression of the aggrecan gene in cartilage by securing binding of Sox 9 to a far-upstream enhancer. Mol. Cell Biol. 28, 4999-5013 (2008).

10. Nikopensius, T. et al. Genetic variants in COL2A1, COL11A2, and IRF6 contribute risk to nonsyndromic cleft palate. Birth Defects Res. A Clin. Mol. Teratol. 88, 748-756 (2010)

11. Melkoniemi, M. et al. Collagen XI sequence variations in nonsyndromic cleft palate, Robin sequence and micrognathia. Eur. J. Hum. Genet. 11, 265-270 (2003).

12. Watanabe, H. et al. Mouse cartilage matrix deficiency ( $\mathrm{cmd}$ ) caused by a $7 \mathrm{bp}$ deletion in the aggrecan gene. Nat. Genet. 7, 154-157 (1994).

13. Tsuda, M., Takahashi, S., Takahashi, Y. \& Asahara, H. Transcriptional co-activators CREB-binding protein and p300 regulate chondrocyte-specific gene expression via association with Sox9. J. Biol. Chem. 278, 27224-27229 (2003).

14. Rau, M. J., Fischer, S. \& Neumann, C. J. Zebrafish Trap230/Med12 is required as a coactivator for Sox9-dependent neural crest, cartilage and ear development. Dev. Biol. 296, 83-93 (2006).

15. Zhou, R. et al. SOX9 interacts with a component of the human thyroid hormone receptor-associated protein complex. Nucleic. Acids Res. 30, 3245-3252 (2002).

16. Hata, K. et al. Paraspeckle protein p54nrb links Sox9-mediated transcription with RNA processing during chondrogenesis in mice. J. Clin. Invest. 118, 3098-3108 (2008).

17. Hattori, T. et al. Transcriptional regulation of chondrogenesis by coactivator Tip60 via chromatin association with Sox9 and Sox5. Nucleic Acids Res. 36, 3011-3024 (2008).

18. Rotin, D. \& Kumar, S. Physiological functions of the HECT family of ubiquitin ligases. Nat. Rev. Mol. Cell Biol. 10, 398-409 (2009).

19. McDonald, F. J. et al. Ubiquitin-protein ligase WWP2 binds to and downregulates the epithelial $\mathrm{Na}(+)$ channel. Am. J. Physiol. Renal. Physiol. 283, F431-436 (2002).

20. Foot, N. J. et al. Regulation of the divalent metal ion transporter DMT1 and iron homeostasis by a ubiquitin-dependent mechanism involving Ndfips and WWP2. Blood 112, 4268-4275 (2008).

21. $\mathrm{Xu}, \mathrm{H}$. M. et al. Wwp2, an E3 ubiquitin ligase that targets transcription factor Oct-4 for ubiquitination. J. Biol. Chem. 279, 23495-23503 (2004).

22. Mertin, S., McDowall, S. G. \& Harley, V. R. The DNA-binding specificity of SOX9 and other SOX proteins. Nucleic Acids Res. 27, 1359-1364 (1999).

23. Yan, Y. L. et al. A pair of Sox: distinct and overlapping functions of zebrafish sox 9 co-orthologs in craniofacial and pectoral fin development. Development 132, 1069-1083 (2005). 
24. Macias, M. J. et al. Structure of the WW domain of a kinase-associated protein complexed with a proline-rich peptide. Nature 382, 646-649 (1996).

25. Yang, F., DeBeaumont, R., Zhou, S. \& Naar, A. M. The activator-recruited cofactor/Mediator coactivator subunit ARC92 is a functionally important target of the VP16 transcriptional activator. Proc. Natl Acad. Sci. USA 101, 2339-2344 (2004).

26. Lee, H. K., Park, U. H., Kim, E. J. \& Um, S. J. MED25 is distinct from TRAP220/MED1 in cooperating with CBP for retinoid receptor activation. EMBO J. 26, 3545-3557 (2007).

27. Leal, A. et al. Identification of the variant Ala335Val of MED25 as responsible for CMT2B2: molecular data, functional studies of the $\mathrm{SH} 3$ recognition motif and correlation between wild-type MED25 and PMP22 RNA levels in CMT1A animal models. Neurogenetics 10, 275-287 (2009).

28. Martin-Serrano, J., Eastman, S. W., Chung, W. \& Bieniasz, P. D. HECT ubiquitin ligases link viral and cellular PPXY motifs to the vacuolar protein-sorting pathway. J. Cell Biol. 168, 89-101 (2005).

29. Murillas, R., Simms, K. S., Hatakeyama, S., Weissman, A. M. \& Kuehn, M. R. Identification of developmentally expressed proteins that functionally interact with Nedd4 ubiquitin ligase. J. Biol. Chem. 277, 2897-2907 (2002).

30. Zou, W. et al. The E3 ubiquitin ligase Wwp2 regulates craniofacial development through mono-ubiquitylation of Goosecoid. Nat. Cell Biol. 13, 59-65 (2010).

31. Rana, R., Surapureddi, S., Kam, W., Ferguson, S. \& Goldstein, J. A. Med25 is required for RNA polymerase II recruitment to specific promoters, thus regulating xenobiotic and lipid metabolism in human liver. Mol. Cell Biol. 31, 466-481 (2011).

32. Akiyama, H. et al. Osteo-chondroprogenitor cells are derived from Sox 9 expressing precursors. Proc. Natl Acad. Sci. USA 102, 14665-14670 (2005).

33. Bi, W., Deng, J. M., Zhang, Z., Behringer, R. R. \& de Crombrugghe, B. Sox9 is required for cartilage formation. Nat. Genet. 22, 85-89 (1999).

34. Walker, M. B. \& Kimmel, C. B. A two-color acid-free cartilage and bone stain for zebrafish larvae. Biotech. Histochem. 82, 23-28 (2007).
35. Lefebvre, V., Huang, W., Harley, V. R., Goodfellow, P. N. \& de Crombrugghe, B. SOX9 is a potent activator of the chondrocyte-specific enhancer of the pro alpha1(II) collagen gene. Mol. Cell Biol. 17, 2336-2346 (1997).

\section{Acknowledgments}

We thank Andreas Schedl, Stephen O'Gorman and James F. Martin for valuable help. These studies were supported by a Grant-in-aid for Scientific Research from the Japanese Ministry of Education, Culture, Sports, Science, and Technology (\#20659231), a Takeda Science Foundation grant to H.A., a National Institutes of Health HD30284 grant to R.R.B. and National Institutes of Health grants HD022486 and DE020076 to J.H.P.

\section{Author contributions}

H.A. directed the study; K.Y., B.O., Y.K., H.M., T.S., N.T. and T.N. performed all molecular and biochemical experiments; J.M.D., Z.Z., R.R.B. and B.d.C. generated mutant mice and performed the GeneChip analysis; and Y.N., X.H., J.H.P. and M.L.W. conducted all in vivo experiments.

\section{Additional information}

Accession codes: The microarray data have been deposited in ArrayExpress under the accession number E-MTAB-537.

Supplementary Information accompanies this paper at http://www.nature.com/ naturecommunications

Competing financial interests: The authors declare no competing financial interests.

Reprints and permission information is available online at http://npg.nature.com/ reprintsandpermissions/

How to cite this article: Nakamura, Y. et al. Wwp2 is essential for palatogenesis mediated by the interaction between Sox9 and mediator subunit 25. Nat. Commun. 2:251 doi: $10.1038 /$ ncomms1242 (2011). 